\section{Multiple antenna aided OFDM employing minimum bit error rate multiuser detection}

\author{
M.Y. Alias, A.K. Samingan, S. Chen and L. Hanzo
}

A minimum bit error rate (MBER) multiuser detector designed for space division multiple access (SDMA) aided orthogonal frequency division multiplexing (OFDM) systems is proposed. It is shown that the MBER detector outperforms the minimum mean squared error (MMSE) detector, since the MBER detector directly minimises the BER, while the MMSE detector minimises the mean-squared error (MSE), which does not guarantee achieving the minimum BER. When supporting two users, the proposed MBER scheme substantially outperforms the classic MMSE arrangement in the investigated propagation scenario.

Introduction: In an effort to increase the achievable system capacity of an OFDM system, antenna arrays can be employed for supporting multiple users in a space division multiple access (SDMA) communications scenario $[1,2]$. A variety of linear multiuser detectors have been proposed for performing the separation of OFDM users based on their unique, user-specific spatial signature, provided that their channel impulse response was accurately estimated [1, 2]. The most popular design strategy is constituted by the minimum mean squared error (MMSE) multiuser detector (MUD). However, as suggested in the context of either conventional channel equalisation [3] or in CDMA [4, 5], a better strategy is to choose the linear detector's coefficients so as to directly minimise the error probability or bit error rate (BER), rather than the mean squared error (MSE), since minimising the MSE does not necessarily guarantee that the BER of the system is also minimised. Detectors that directly minimise the BER are referred to as minimum-BER (MBER) detectors [3-5]. Against this background, in this Letter, we contrive the MBER linear MUD designed for the uplink of an SDMA/OFDM system.

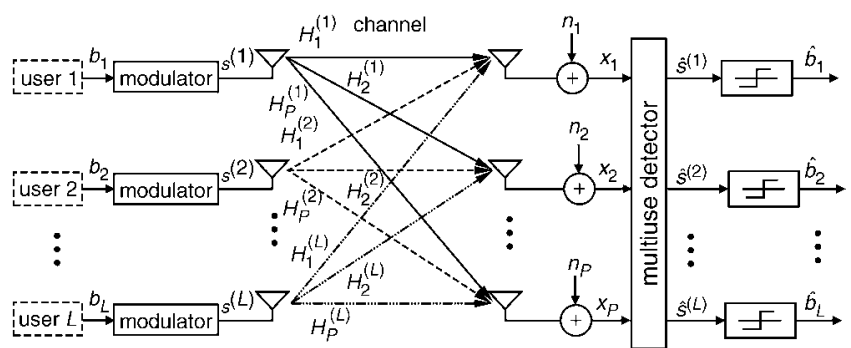

Fig. 1 Schematic diagram of antenna array aided OFDM uplink scenario Each $L$ user equipped with single transmit antenna and BS receiver assisted by $P$-element antenna front end

SDMA system model: The SDMA OFDM system is capable of differentiating $L$ users' transmitted signals at the base station (BS) invoking their unique, user-specific spatial signature created by the channel transfer functions or channel impulse responses (CIR) between the users' single transmit antenna and the $P$ different receiver antennas at the BS [2]. Fig. 1 portrays the antenna array aided uplink transmission scenario considered. In this Figure, each of the $L$ simultaneous users is equipped with a single transmission antenna, while the receiver capitalises on a $P$-element antenna front end. The set of complex signals, $x_{p}[n, k], p \in 1, \ldots, P$ received by the $P$ element antenna array in the $k$ th subcarrier of the $n$th OFDM symbol is constituted by the superposition of the independently faded signals associated with $L$ users sharing the same space-frequency resource [2]. The received signal was corrupted by Gaussian noise at the array elements. The indices $[n, k]$ have been omitted for notational convenience during our forthcoming discourse, yielding [2]:

$$
\mathbf{x}=\mathbf{H s}+\mathbf{n}=\overline{\mathbf{x}}+\mathbf{n}
$$

where $\mathbf{x}$ is the $(P \times 1)$-dimensional vector of the received signals, $\mathbf{s}$ is the $(L \times 1)$-dimensional vector of the transmitted signals, $\mathbf{n}$ is the $(P \times 1)$-dimensional noise vector and $\overline{\mathbf{x}}$ represents the noiseless component of $\mathbf{x}$. The complex data signal, $s_{l}$, transmitted by the $l$ th user, $l \in 1, \ldots, L$ and the AWGN noise process, $n_{p}$, at any antenna array element $p, p \in 1, \ldots, P$ are assumed to exhibit a zero mean and a variance of $\sigma_{l}^{2}$ and $\sigma_{n}^{2}$ for the data signal and AWGN noise process, respectively. The frequency domain channel transfer function (FDCHTF) matrix $\mathbf{H}$ of dimension $P \times L$ is constituted by the set of channel transfer function vectors of the $L$ users, each of which describes the FDCHTF between the single transmitter antenna associated with a particular user $l$ and the reception array elements $p \in 1, \ldots, P$. The FDCHTFs, $H_{p l}$ of the different array elements $p \in 1, \ldots, P$ for users $l \in 1, \ldots, L$ are independent, stationary, and complex Gaussian distributed processes with zero mean and unit variance. For linear MUDs, the estimate $\hat{\mathbf{s}}$ of the transmitted signal vector $\mathbf{s}$ of the $L$ simultaneous users is generated by linearly combining the signals received by the $P$ different antenna elements at the BS with the aid of the array weight matrix W, resulting in $\hat{\mathbf{s}}=\mathbf{W}^{H} \mathbf{x}$. By substituting $\mathbf{x}$ with (1) and considering the $l$ th user's associated vector component, we arrive at: $\hat{s}_{l}=\mathbf{w}_{l}^{H} \mathbf{x}=\mathbf{w}_{l}^{H} \mathbf{H s}+\mathbf{w}_{l}^{H} \mathbf{n}=\overline{\mathbf{s}}_{l}+\mathbf{w}_{l}^{H} \mathbf{n}$, where the weight vector $\mathbf{w}_{l}$ is the $l$ th column of the weight matrix $\mathbf{W}$. At the current state-of-the-art, the most popular MUD strategy is the MMSE design, where $\mathbf{w}_{l}$ is chosen as the unique vector minimising the MSE expressed as MSE $=$ $E\left[\left(\hat{s}_{l}-s_{l}\right)^{2}\right]$, namely as [2] $\mathbf{w}_{l(M M S E)}=\left(\mathbf{H H}^{H}+\sigma_{n}^{2} \mathbf{I}\right)^{-1} \mathbf{H}_{l}$, where $\mathbf{H}_{l}$ is the $l$ th column of the FDCHTF matrix $\mathbf{H}$.
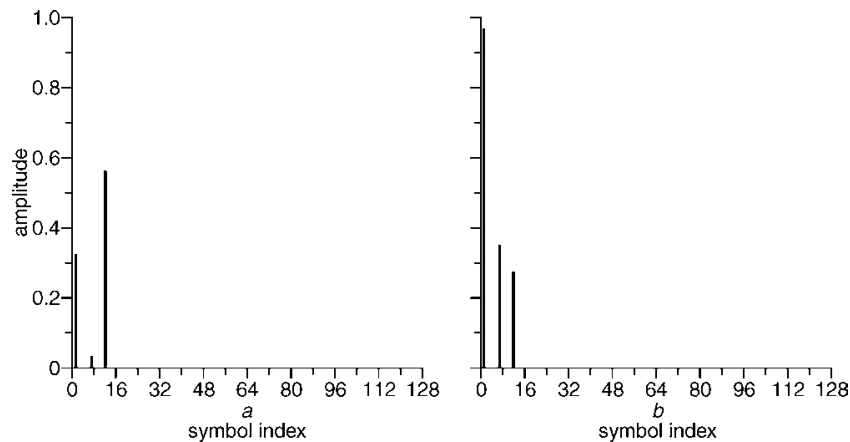

Fig. 2 Two different channel impulse responses (CIR) recorded at two BS receiver antennas for user 1; another pair of different CIRs experienced by user 2

$a$ CIR 1: user 1, antenna $1 \quad b$ CIR 2: user 1, antenna 2

Error probability in BPSK system: In this Letter the term BER and probability of error $P_{E}$ are used interchangeably. The BER encountered at the output of the MUD $\mathbf{w}_{l}$ of user $l$ may be expressed as [5] $P_{E}\left(\mathbf{w}_{l}\right)=\left(1 / N_{b}\right) \sum_{j=1}^{N_{b}} Q\left[c_{j}\left(\mathbf{w}_{l}\right)\right]$, where $c_{j}\left(\mathbf{w}_{l}\right)$ is given by:

$$
c_{j}\left(\mathbf{w}_{l}\right)=\frac{\operatorname{sgn}\left(b_{l}^{(j)}\right) \cdot \bar{s}_{l}^{(j)}}{\sigma_{n} \sqrt{\mathbf{w}_{l}^{H} \mathbf{w}}}=\frac{\operatorname{sgn}\left(b_{l}^{(j)}\right) \cdot \mathbf{w}_{l}^{H} \overline{\mathbf{x}}_{j}}{\sigma_{n} \sqrt{\mathbf{w}_{l}^{H} \mathbf{w}}}
$$

and $\overline{\mathbf{x}}_{j}, j \in 1, \ldots, N_{b}$, constitutes a possible value of $\overline{\mathbf{x}}$ defined in the context of (1). It may be shown that the BER is invariant to a positive scaling of the weight vector, i.e. the BER depends only on the vectorial direction of $\mathbf{w}_{l}$, but not on its magnitude.

Exact MBER (EMBER) multiuser detection: The MBER solution is defined as [5] $\mathbf{w}_{l(M B E R)}=\arg \min _{\mathbf{w}_{l}} P_{E}\left(\mathbf{w}_{l}\right)$. However, the complex, irregular shape of the BER cost function prevents us from deriving a closed-form solution for the MBER OFDM/SDMA MUD weights. Therefore in practice an iterative strategy based on the steepestdescent gradient method can be used for finding the MBER solution [5]. According to this method, the linear MUD's weight vector $\mathbf{w}_{l}$ is iteratively updated, commencing for example from the MMSE weights, until the weight vector that exhibits the lowest BER is arrived at. In each step, the weight vector is updated according to a specific step size, $\mu$, in the vectorial direction in which the BER cost function decreases most rapidly, namely in the direction opposite to the gradient of the BER cost function, which is given by:

$$
\begin{aligned}
\nabla_{\mathbf{w}_{l}} P_{E}\left(\mathbf{w}_{l}\right)= & \frac{1}{N_{b} \sqrt{2 \pi} \sigma_{n}}\left(\frac{\mathbf{w}_{l} \mathbf{w}_{l}^{H}-\mathbf{w}_{l}^{H} \mathbf{w}_{l} \mathbf{I}}{\left(\mathbf{w}_{l}^{H} \mathbf{w}_{l}\right)^{3 / 2}}\right) \\
& \times \sum_{j=1}^{N_{b}} e^{\left(-\left(\bar{s}_{l}^{(j)}\right)^{2} / 2 \sigma_{n}^{2} \mathbf{w}_{l}^{H} \mathbf{w}_{l}\right)} \cdot \operatorname{sgn}\left(b_{l}^{(j)}\right) \cdot \overline{\mathbf{x}}_{j}
\end{aligned}
$$

Observe in (2) that BER is independent of the magnitude of the MUD's weight vector, and the knowledge of the orientation of the detector's weight vector is sufficient for defining the decision boundary of the 
linear MBER OFDM/SDMA detector. Therefore the MBER detector has an infinite number of solutions, although with the aid of appropriate weight-vector normalisation it is possible to reduce the infinite number of MBER solutions to a single solution. In our previous discourse we assumed the explicit knowledge of the FDCHTF matrix $\mathbf{H}$ defined in (1). However, in practice $\mathbf{H}$ has to be determined on the basis of the channel impaired noisy value of $\mathbf{x}$ and hence a number of adaptive techniques have been proposed in [3-5] to this effect, which are also applicable to OFDM/SDMA.

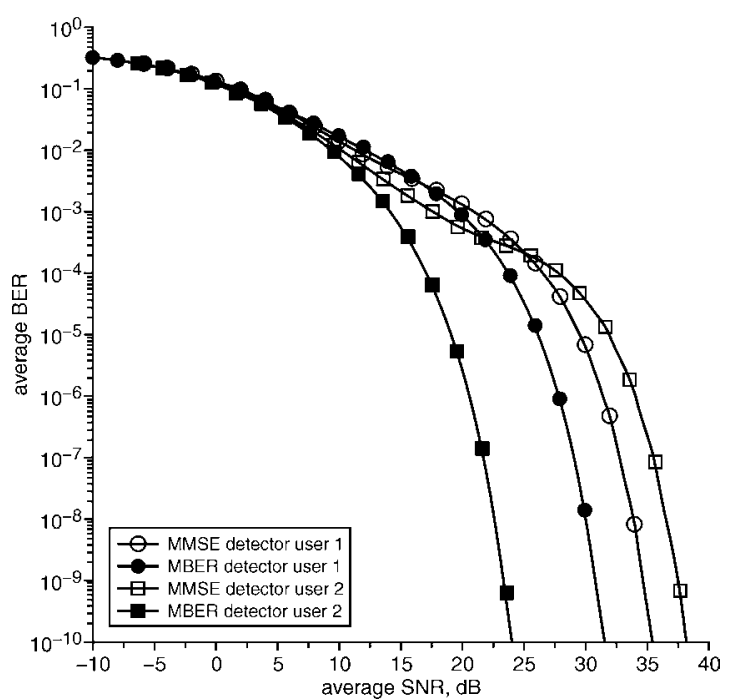

Fig. 3 Average BER against average SNR for MMSE and MBER multiuser detectors of user 1 and user 2 supported by two receiver antennas using 128 subcarrier OFDM communicating over channel characterised by CIRs shown in Fig. 2

Simulation results: In our quantitative investigations we used the simplest possible SDMA OFDM system supporting two users with the aid of two receiver antennas. As shown in Fig. 1, each user has a unique channel impulse response (CIR) with respect to each receiver antenna. The two CIRs corresponding to user 1 and the two BS antennas are shown in Fig. 2. The CIRs represent a three-path indoor type channel [2], where no fading is experienced. The OFDM modem had 128 subcarriers. In our simulations, we initialised the iterative MBER algorithm to the MMSE MUD weights. The average BER of user 1 and user 2 recorded in the context of both the MMSE and MBER detector is shown in Fig. 3. We can see from this Figure that user 1 has a better average BER in conjunction with the MMSE detector compared to user 2 for SNRs in excess of about $25 \mathrm{~dB}$. By contrast, the MBER detector of user 2 outperforms that of user 1 in terms of the average BER. We can also see that the MBER detectors of both users exhibit a substantially lower average BER compared to the MMSE detectors. Again, as expected, this is because the MMSE MUD is directly minimising the MSE and not the BER. We may also note that the average BER difference between the MMSE and MBER detectors is not the same for both users. Specifically, the MBER MUD of user 2 has an SNR advantage of almost $12 \mathrm{~dB}$, while that of user 1 has about $5 \mathrm{~dB}$ SNR advantage. This is a consequence of the unique combinations of the CIRs of both users, since it can be seen in Fig. 2 that the CIR of user 1 exhibits a lower ratio between the main and the delayed CIR taps than that of user 2 , hence receiving less useful signal energy and more multipath interference. To conclude, the MBER SDMA/OFDM MUD is capable of providing a substantial performance gain.

(C) IEE 2003

26 August 2003

Electronics Letters Online No: 20031105

DOI: 10.1049/el:20031105

M.Y. Alias, A.K. Samingan, S. Chen and L. Hanzo (School of ECS, University of Southampton, Southampton SO17 1BJ, United Kingdom)

E-mail: 1h@ecs.soton.ac.uk

\section{References}

1 VANDENAMEELE, P., VAN DER PERRE, L., ENGELS, M.G.E., GYSELINCKX, B., and DE MAN, H.J.: 'A combined OFDM/SDMA approach', IEEE J. Sel. Areas Commun., 2000, 18, (11), pp. 2312-2321

2 HANZO, L., MÜNSTER, M., CHOI, B.J., and KELLER, T.: 'OFDM and MC-CDMA' (John Wiley and IEEE Press, UK, 2003)

3 YEH, C.-C., and BARRY, J.R.: 'Adaptive minimum bit-error rate equalization for binary signalling', IEEE Trans. Commun., 2000, 48, (7), pp. $1226-1235$

4 WANG, X., LU, W.-S., and ANTONIOU, A.: 'Constrained minimum-BER multiuser detection', IEEE Trans. Signal Process., 2000, 48, (10), pp. 2903-2909

5 CHEN, S., SAMINGAN, A.K., MUlgRew, B., and HANZO, L.: 'Adaptive minimum-BER linear multiuser detection for DS-CDMA signals in multipath channels', IEEE Trans. Signal Process., 2001, 49, pp. $1240-1247$ 